\title{
A Potentially Stabilizing Magnetic Effect in Astrophysical Jets
}

\author{
M. Viallet and H. Baty \\ Observatoire astronomique, Strasbourg, FRANCE \\ email: viallet@astro.u-strasbg.fr \\ email: baty@astro.u-strasbg.fr
}

\begin{abstract}
We investigate the development of Kelvin-Helmholtz instabilities in magnetized jets by means of high-resolution spatial simulations. Our main finding is the existence of a stabilizing mechanism driven by the initial disruption of the jet. This mechanism gives rise to a local amplification of the magnetic field, which in turn leads to an enhanced stability of the jet configuration.
\end{abstract}

Keywords. Instabilities - magnetohydrodynamics (MHD) - ISM : jets and outflows - methods : numerical - plasmas

\section{Introduction}

There is a well established discrepancy between observations of long well collimated jets and numerical simulations. Indeed, the later predict that jets are quickly disrupted due to the development of internal magnetohydrodynamic (MHD) instabilities. In particular, the Kelvin-Helmholtz (KH) instability is known to be the most dangerous for the jet collimation (see reviews by Birkinshaw 1991, Ferrari 1998).

In this work we reinvestigate the development and evolution of the $\mathrm{KH}$ in a weakly magnetized jet. We use a spatial approach, where we follow the development of the KH instability from an initial perturbation introduced at the inlet of the numerical domain.

\section{Setup}

We consider a two-dimensional jet embedded in an uniform axial magnetic field. As we are interested in the weak magnetic field regime, relevant for astrophysical jets, we consider Alfénic Mach number $M_{A}=V_{0} / V_{A}$ in the range $[5 \rightarrow 15]$. We focus mainly on the transonic regime of the jet $\left(M_{s}=1\right)$. Indeed, before reaching the asymptotic supersonic regime, the jet is accelerated in an intermediate region where it must survive instabilities.

We use the modern shock-capturing code VAC (see Tóth 1996) to solve the ideal MHD equations with a TVD scheme based on Roe approximate Riemann solver. We use domain size of $40 \times 16$ and $80 \times 32$ jet's diameter and a typical resolution of $1200 \times 480$ grid points. The jet is injected at the left boundary of the numerical domain, where a perturbation of small amplitude is applied to drive the $\mathrm{KH}$ modes unstable.

\section{Results}

Our simulations show that the development of the KH instability first leads to a largescale disruption of the jet (see fig. 1). The first disruption happens at approximatively 40 jet's radii independently from the initial $M_{A}$. This result is in good agreement with 


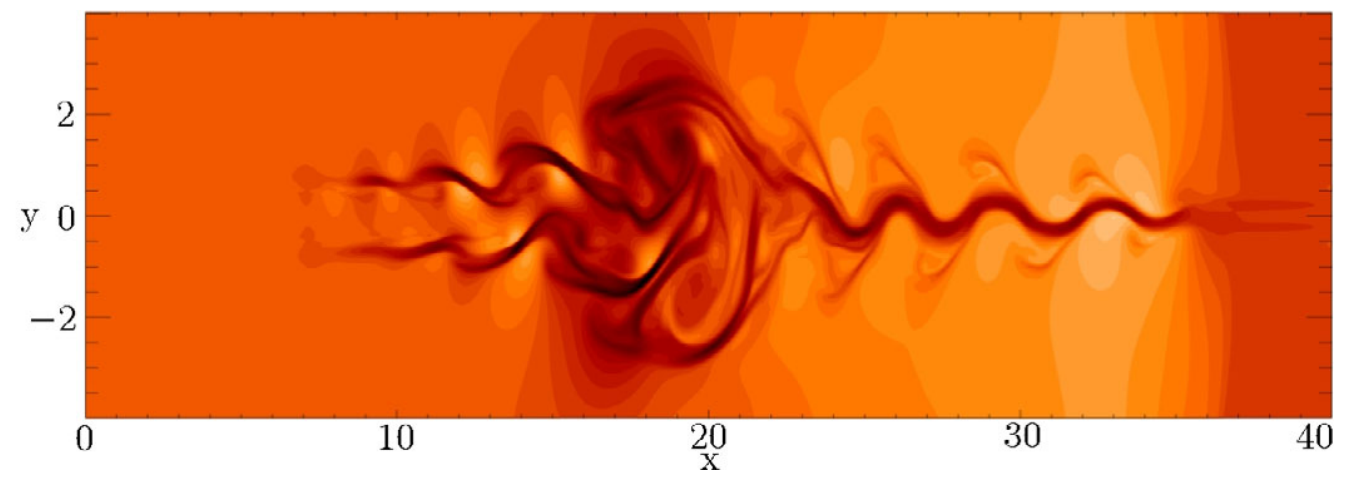

Figure 1. Snapshot in density contrast of the first disruption of the jet. The jet travels from left to right. Behind the disruption front at $x=20$, a trail at $x=8$ is propagating upstream. It is associated with a local amplification of the magnetic field.

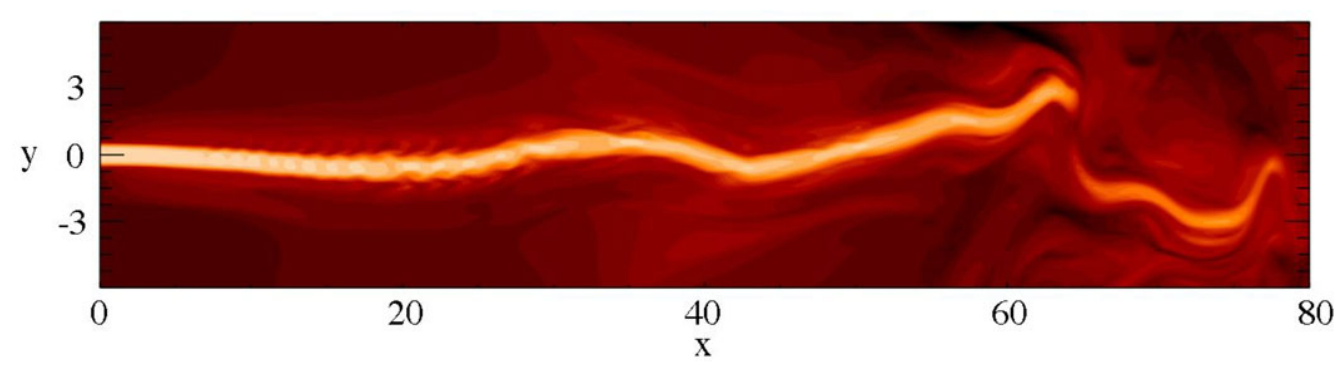

Figure 2. Snapshot in axial velocity contrast of a self-stabilized jet, a long time after the first disruption. In comparison with fig. 1 , the jet now reach $x=60$ while keeping a good collimation.

previous studies, in particular with temporal studies (see, e.g., Baty \& Keppens 2006) which assume periodic boundary conditions thus neglecting possible convective effects.

However our spatial simulations suggest that this first disruption is actually a transient phenonemon. Indeed, in Figure 1 one can notice a trail structure at $x=8$. This trail is actually associated with an amplification of the magnetic field (not shown here) on both sides of the jet that is propagating upstream. When this trail reaches the left boundary, it results an enhanced stability of the jet. This is for example the case in Figure 2, showing a snapshot of the jet a long time after the first disruption. The jet is now able to reach $x=60$ without losing its collimation. This mechanism is studied in more detail in Viallet \& Baty (in preparation).

\section{Conclusions}

In agreement with previous studies we show that an unstable jet is quickly disrupted by the development of the KH instability. However, our spatial study suggests the existence of a "self-stabilizing" mechanism. Indeed the first disruption drives a local amplification of the magnetic field on both sides of the jet which has a stabilizing effect. Such selfstabilizing mechanisms could be an important ingredient to explain the apparent stability of astrophysical jets and their numerical study requires the use of a spatial approach. 


\section{References}

Baty, H. \& Keppens, R. 2006, A\&A, 447, 9

Birkinshaw, M. 1991, in Beams and Jets in Astrophysics, Cambridge Univ. Press, 279

Ferrari, A. 1998, Annual Rev. Astrophys., 36, 539

Viallet, M. \& Baty, H. in preparation

Tóth, G. 1996, Astrophysical Letters \& Communications, 34, 245 